\title{
Engineering bacteria to monitor the bleeding of animals using far-red
}

fluorescence

Zi-Zhu Tan ${ }^{1}$, Xiao-Dan Li ${ }^{1}$, Chao-Di Kong ${ }^{1}$, Na Sha ${ }^{1}$, Ya-Nan Hou ${ }^{1}$, Kai-Hong Zhao $1, *$

${ }^{1}$ State Key Laboratory of Agricultural Microbiology, Huazhong Agricultural University, Wuhan 430070, P.R. China;

Corresponding author: Kai-Hong Zhao, State Key Laboratory of Agricultural Microbiology, Huazhong Agricultural University, Wuhan 430070, P.R. China, E-mail: khzhao@163.com

\section{Supplementary Materials}

(7 Supplementary Figures, 2 Supplementary Tables) 


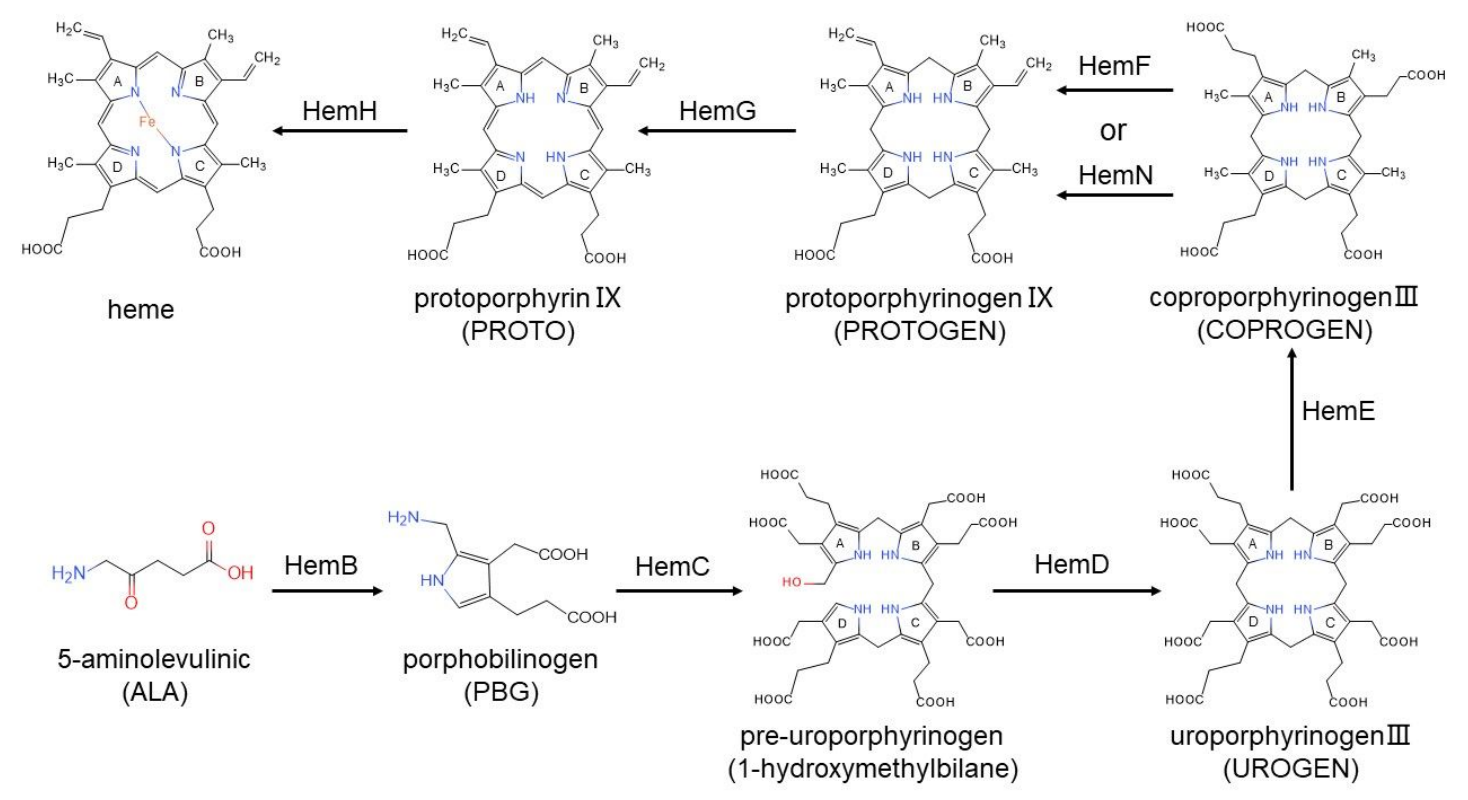

Figure S1. Scheme of heme formation in E. coli. Biosynthesis of a heme starts with the synthesis of 5-aminolevulinic acid (5-ALA). Then, the first cyclic tetrapyrrole uroporphyrinogen III is formed from the precursor 5-ALA in three enzymatic steps (catalyzed by HemB, C and D) via the intermediates: porphobilinogen and pre-uroporphyrinogen. Uroporphyrinogen III is converted into heme in four consecutive enzymatic steps (catalyzed by $\operatorname{HemE}, F(N), G$ and $H$ ) via the intermediates: coproporphyrinogen III, protoporphyrinogen IX, and protoporphyrin IX. In the antepenult step of heme biosynthesis, coproporphyrinogen III is oxidized yielding protoporphyrinogen IX by either oxygen-dependent coproporphyrinogen III oxidase (HemF) or oxygen-independent coproporphyrinogen III oxidase (HemN) ${ }^{1,2}$. Chemical formulas were created with InDraw (5.1.0). 

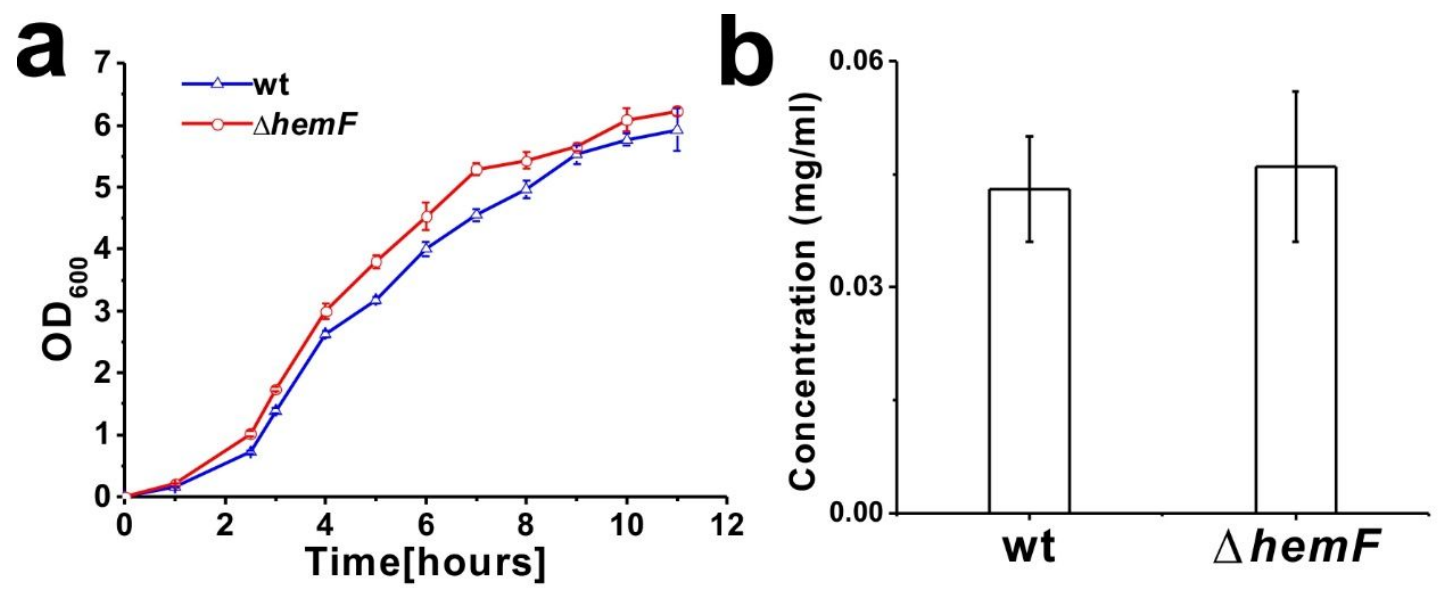

Figure S2. Comparisons between $E$. coli and the mutant with the deletion of hemF. (a) Time-dependent E. coli growth curves in $100 \mathrm{ml}$ LB medium with the shaker of 150 revolutions per minute $(\mathrm{rpm})$ at $37^{\circ} \mathrm{C} . \mathrm{OD}_{600}$ was used to measure the concentration of E. coli. (b) E. coli and the mutant lacking hemF were used to express BDFP1.6 with the induction of IPTG for $14-16$ hours at $16{ }^{\circ} \mathrm{C}$. Protein concentrations were obtained by measuring absorbance at $280 \mathrm{~nm}$. The molar extinction coefficient of BDFP1.6 at $280 \mathrm{~nm}$ was predicted as $17460 \mathrm{~mol}^{-1} \mathrm{~cm}^{-1}$. Error bars show standard deviation $(n=3)$. 

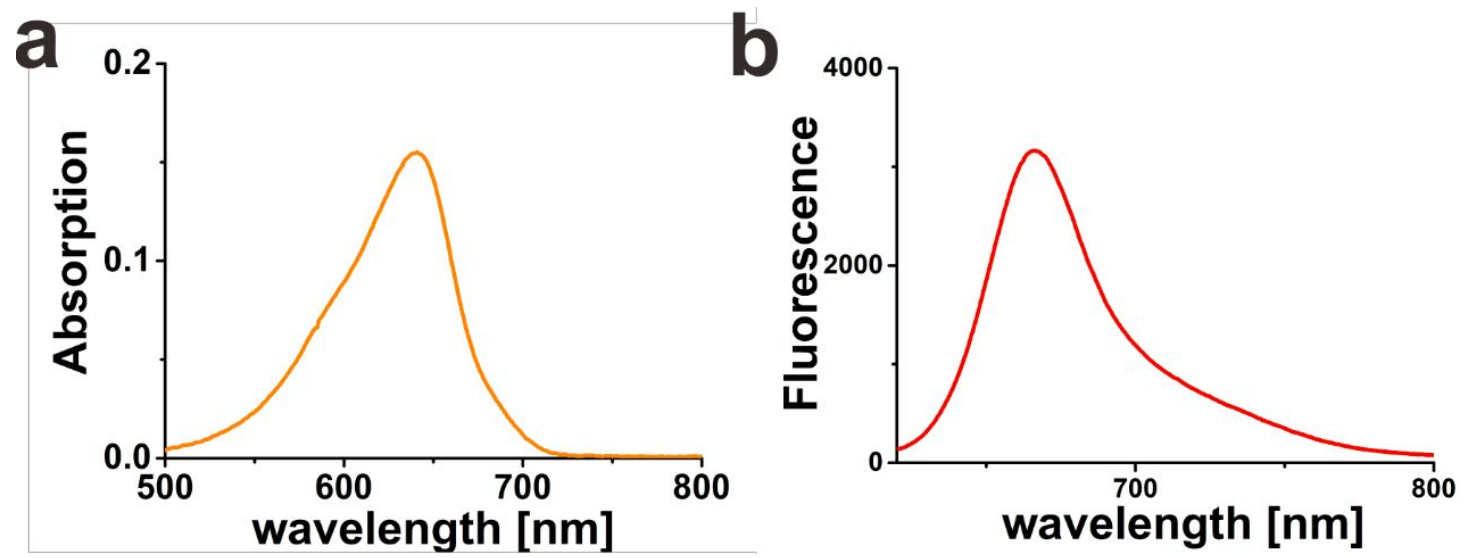

Figure S3. The spectra of BDFP1.6. Absorption (a) and fluorescence emission spectra (b, $\lambda_{\mathrm{ex}}=600 \mathrm{~nm}$ ) of BDFP1.6 in $20 \mathrm{mM} \mathrm{KPB}$ buffer containing $500 \mathrm{mM} \mathrm{NaCl}$ $(\mathrm{pH}=7.2)$. For their chromophorylation, the plasmids (Table S2) for generating BDFP1.6 and the plasmid (Table S2) for generating BV were transformed into $E$. coli cells. After induction and expression, the cells containing the chromophorylated BDFP1.6 were isolated (see Material and methods). Protein samples were purified by $\mathrm{Ni}^{2+}$ affinity chromatography and then changed to $20 \mathrm{mM} \mathrm{KPB}$ buffer containing 500 $\mathrm{mM} \mathrm{NaCl}(\mathrm{pH} 7.2)$ by dialysis. 

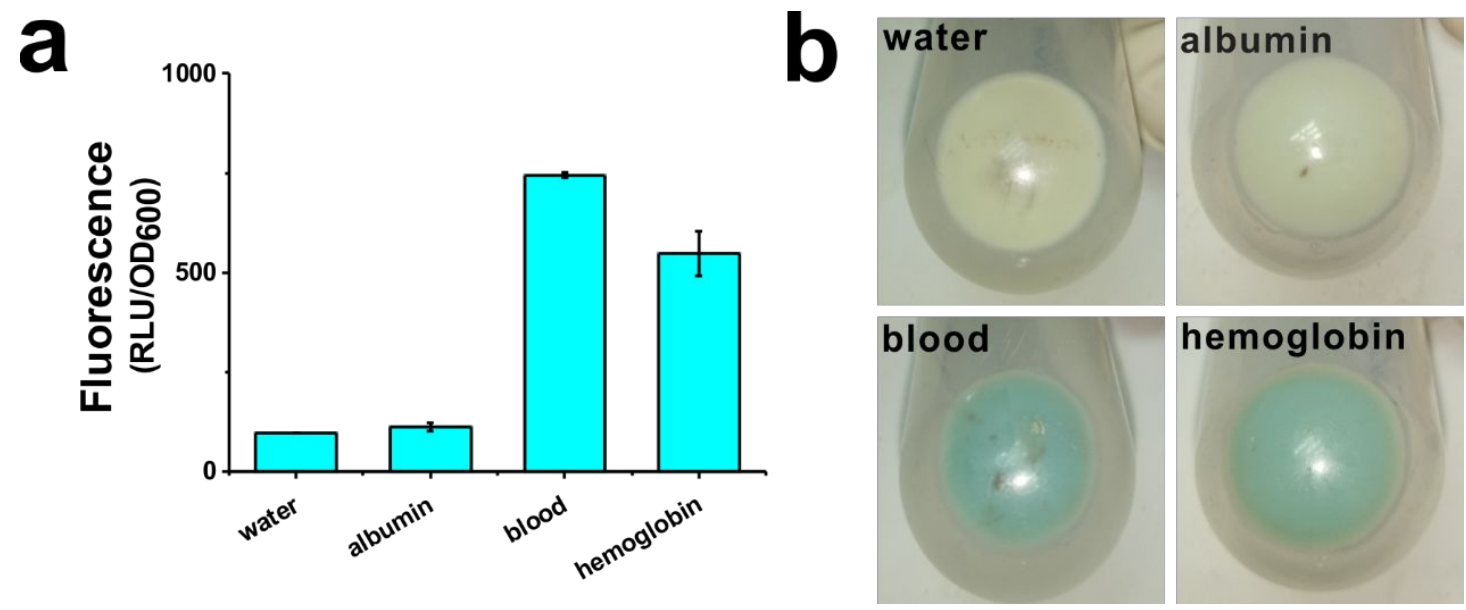

Figure S4. Influence of blood and its components on activation of FRLBD

fluorescence. The fluorescence intensity $\left(\lambda_{\mathrm{ex}}=600 \mathrm{~nm}, \lambda_{\mathrm{em}}=620 \mathrm{~nm}\right)(\mathrm{a})$ and cells colors (b) in the FRLBD with $10 \mu \mathrm{M}$ albumin, $10 \mu \mathrm{M}$ hemoglobin, $1000 \mathrm{ppm}$ blood and water. Blood and hemoglobin could activate the fluorescence of FRLBD. All data has been subtracted from the background fluorescence of the cells not expressing BDFP1.6. Error bars show standard deviation $(n=3)$. 

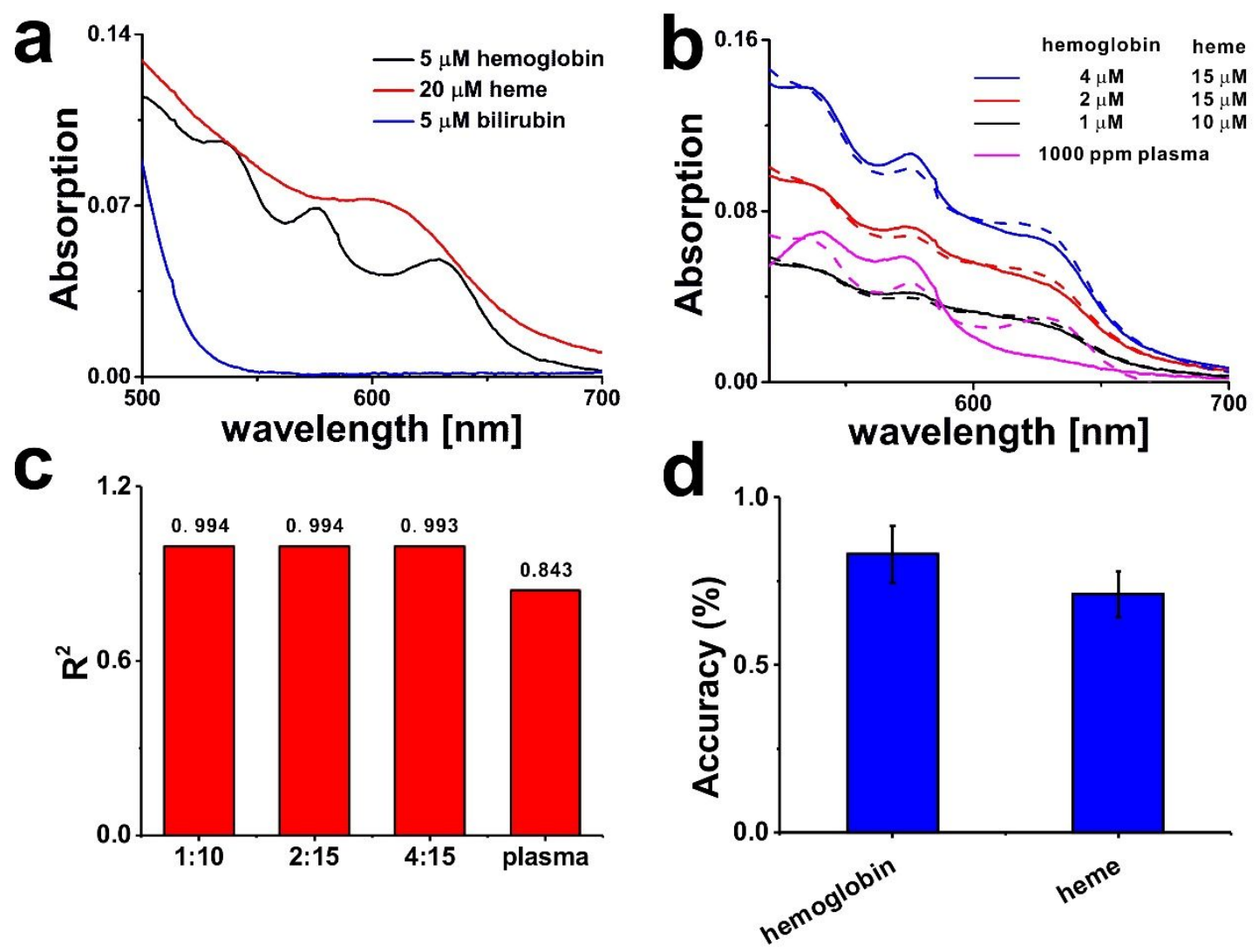

Figure S5. Determination of heme and hemoglobin in blood. (a) Standard spectra of hemoglobin, heme and bilirubin in $20 \mathrm{mM} \mathrm{KPB} \mathrm{(pH} \mathrm{=} \mathrm{7.2).} \mathrm{(b)} \mathrm{Absorption} \mathrm{spectra}$ (solid lines) of 1000 ppm plasma and different concentrations of heme and hemoglobin in $20 \mathrm{mM} \mathrm{KPB}(\mathrm{pH}=7.2)$. Dashed lines showed the result of multiple linear regression using hemoglobin, heme and bilirubin between 520 and $700 \mathrm{~nm}$. (c) Evaluation (R2) of multiple linear regressions. The closer it is to 1, the better the fitting effect. (d) Accuracy of heme and hemoglobin calculated using this method (accuracy $\left.=1-\frac{\left|C_{t}-C_{e}\right|}{C_{t}}\right) \cdot \mathrm{C}_{\mathrm{t}}$ and $\mathrm{C}_{\mathrm{e}}$ represent the true concentration and the estimated concentration, respectively. Error bars show standard deviation $(n=3)$. 

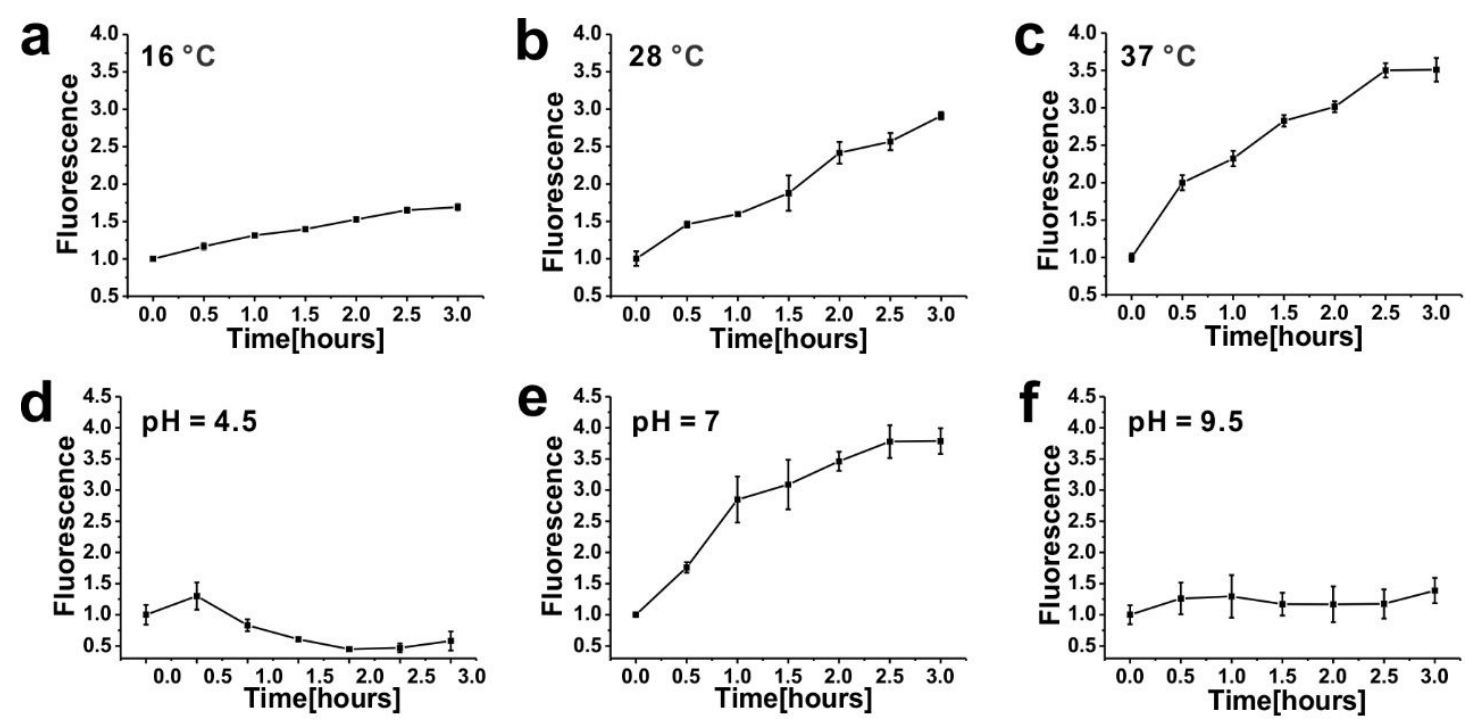

Figure S6. Reaction conditions of FRLBD with blood in vitro. Engineered E. coli lacking hemF but expressing HO1, BDFP1.6 and ChuA induced by IPTG for 14-16 hours at $16{ }^{\circ} \mathrm{C}$ was exposed to $1000 \mathrm{ppm}$ blood in different temperature (a,b and c) or $\mathrm{pH}(\mathrm{d}, \mathrm{e}$, and $\mathrm{f})$. Fluorescence was measured every 30 minutes. Error bars denote the SD for three independent biological replicates conducted with the different $E$. coli. All data has been subtracted from the background fluorescence of the cells not expressing BDFP1.6. 
a

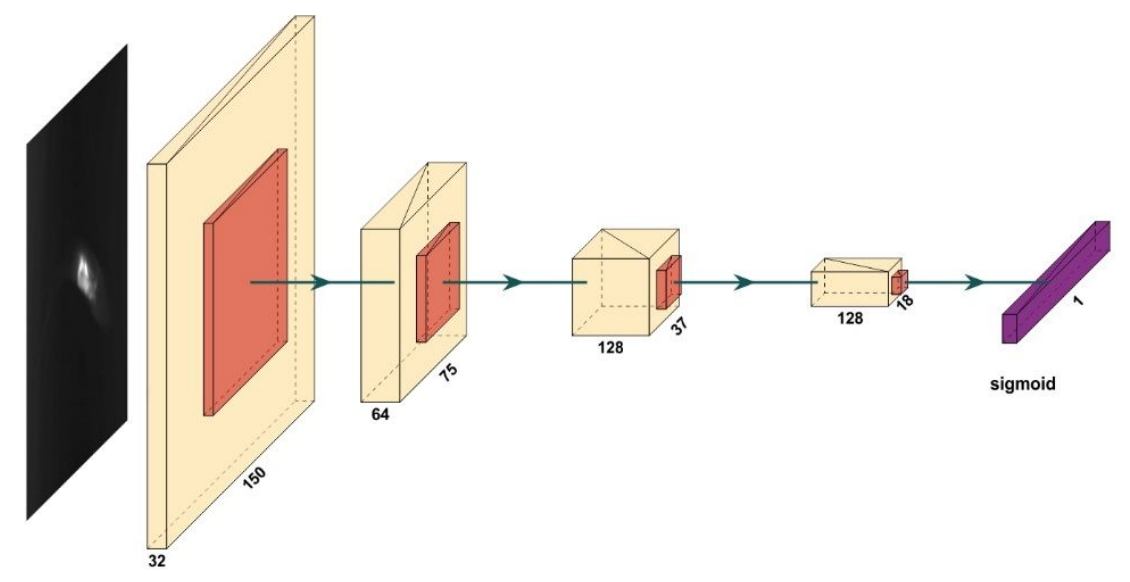

b

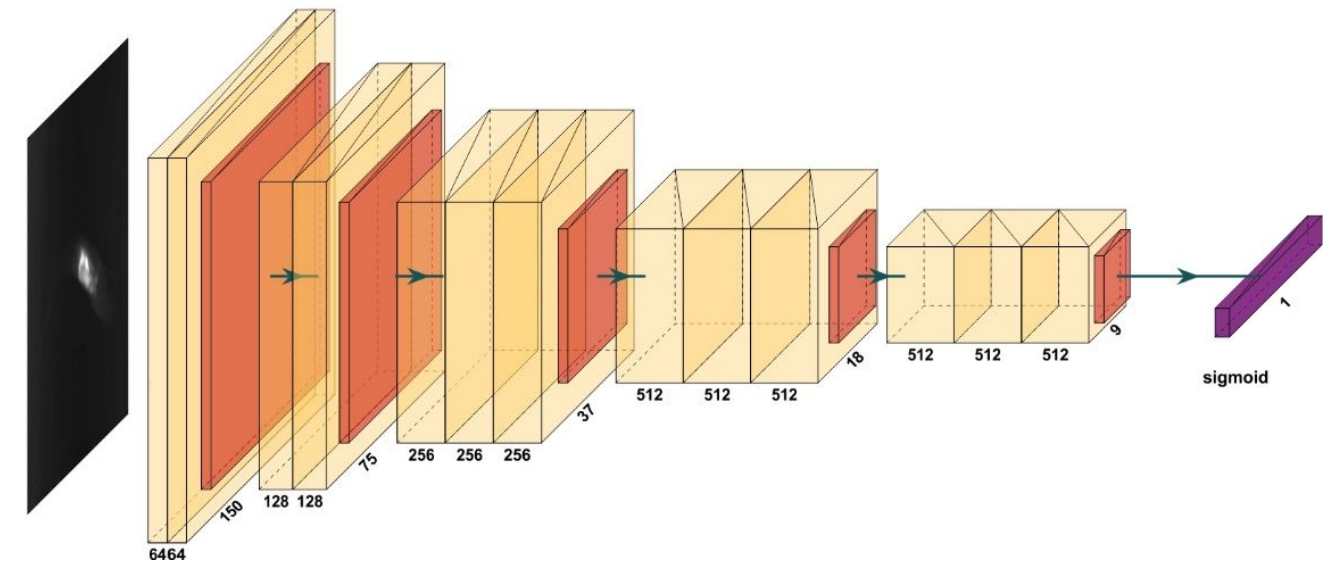

Figure S7. Models of CNNs. The models of a simple CNN containing 4 Conv2D and 4 MaxPooling2D layers (a) and VGG16 (b). Yellow and red represent the Conv2D layers and MaxPooling2D layers, respectively. In the last steps, sigmoid function was used to determine the probability of images belong to the bleeding zebrafish. The number represent shapes of the Conv2D layers. Figure was created using PlotNeuralNet (https://github.com/Harislqbal88/PlotNeuralNet). 
Table S1: PCR Primers for the construction of gene circle

\begin{tabular}{lcl}
\hline Primer & \multicolumn{1}{c}{ Sequence } & clone \\
\hline P1 & 5'- GGCATCCCTCTGGGTCCCACTGCCCGGGGC -3' & pACYCDuet-chuA \\
P2 & 5'- GAGGGAGTTGAATGTATCCCGCAGCCCGGC -3' & \\
P3 & 5'- CCTGGCGGGTCGGCCTATACAACAA -3' & \\
P4 & 5'- GGACACCAACTCTGGGTACTCCTGA -3' & pCDFDuet-ho1 \\
P5 & 5'- GACCTCAACTACTTCCTCAGGATGTGT -3' & \\
P6 & 5'- CCTCACACACATGTTGTAGCGCCTTGT -3' & pCDFDuet-ho1-bdfp1.6 \\
\hline
\end{tabular}


Table S2. Plasmids used for the construction of gene circle and deletion of hemF

\begin{tabular}{ll}
\hline Antibiotic resistance & Plasmids \\
\hline Kanamycin & pKD4 \\
Ampicillin & pKD46 \\
streptomycin & pCDFDuet-ho1-bdfp1.6 \\
chloromycetin & pACYCDuet-chuA \\
\hline
\end{tabular}




\section{Reference}

1. Layer, G.; Reichelt, J.; Jahn, D.; Heinz, D. W., Structure and function of enzymes in heme biosynthesis. Protein Sci. 2010, 19 (6), 1137-1161.

2. Heinemann, I. U.; Jahn, M.; Jahn, D., The biochemistry of heme biosynthesis. Arch. Biochem. Biophys. 2008, 474 (2), 238-251. 